\title{
Caminhos formacionais na Engenharia de Produção: uma análise do conteúdo das provas específicas do ENADE nos anos 2005,2008 e 2011
}

Flaviane Rodrigues de Sousa

flaviane_rs@hotmail.com

Daiana Medeiros da Silva

daiana.medeirosdasilva@gmail.com

José André Villas Bôas Mello

joseavbm@yahoo.com.br

Andréa Justino Ribeiro Mello

andreajribeiro@yahoo.com.br

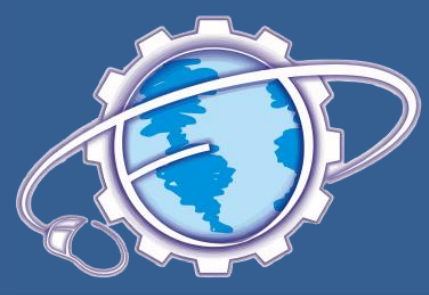

\section{RESUMO}

O objetivo deste artigo é apresentar uma análise de provas do ENADE (Exame Nacional de Desempenho de Estudantes) do grupo VI de Engenharia, na busca de evidências sobre os conteúdos mais exigidos dos futuros profissionais da área de Engenharia de Produção. A análise realizada está embasada na revisão da literatura e na comparação das diretrizes propostas pela ABEPRO e pelo manual do ENADE. Foram observadas, a partir de um estudo detalhado, as provas específicas de Engenharia de Produção aplicadas nos anos de 2005, 2008 e 2011; averiguando-se um total de 67 questões. Como resultado geral pode-se perceber que ao longo do período analisado não há áreas da Engenharia de Produção que tenham uma representatividade muito superior às outras. Por outro lado, algumas delas se destacam por serem pouco contempladas na totalidade das questões. Outro aspecto observado é que não há uma constância sobre o conhecimento exigido nos anos estudados, mas sim uma variação do quantitativo de questões por área por ano de aplicação da prova.

Palavras-chave: ENADE; Educação em Engenharia; Engenharia de Produção.

\section{Formational paths in Production Engineering: an analysis in the content of the ENADE's specific exams in the years 2005, 2008 and 2011}

\begin{abstract}
The objective of this paper is to present an analysis of the ENADE's (National Examination Performance of Students) tests of the VI Engineering group, searching for evidence about the content which is the most required of future professionals in the area of Production Engineering. The analysis is based on a literature review and on the comparison of the guidelines proposed by ABEPRO and by the ENADE's manual.Under review, through a detailed study, in the specific tests of Production Engineering applied in 2005, 2008, and 2011; containing a total of 67 questions. As a general result, it shows that in the period analyzed there are no areas of Production Engineering which present a much higher representation than the others. On the other hand, some of the questions stand out because they are less contemplated in the total number of the questions. Another point observed is that there isn't constancy about the knowledge required in the years that were anlyzed, but there is a variation of quantitative questions per area and per year of the test's application.
\end{abstract}

Keywords: ENADE; Engineering Education; Production Engineering. 


\section{Considerações iniciais}

Esse trabalho é fruto de um desdobramento do artigo: "Caminhos formacionais na Engenharia de Produção: evidências sobre demandas das organizações públicas", o qual foi publicado e apresentado no III Encontro Fluminense de Engenharia de Produção (EnfePro/2011) que procurou identificar os conteúdos formacionais inseridos nas provas de acesso das organizações públicas, relacionando-os com as respectivas áreas de conhecimento de um curso de Engenharia de Produção. Acredita-se que o diagnóstico de conteúdo das provas de seleção que se dediquem a selecionar e avaliar egressos dos cursos de engenharia de produção possa contribuir para a organização de um curso voltado a demandas de mercado, principalmente, para um curso de bacharelado que passa por constante processo evolutivo tanto no eixo técnico quanto no eixo de gestão.

Apesar do curso de Engenharia de Produção ser um dos mais recentes da área de Engenharia, ainda assim, é um dos que apresenta maior crescimento na atualidade. E um dos elementos que contribui para explicar este fator é a evolução do mundo da produção. $\mathrm{O}$ desenvolvimento que vem ocorrendo recentemente determina que um dos aspectos mais importantes das empresas atuais é o conhecimento, e este influencia diretamente nas estratégias de competição em processos produtivos, qualidade, além de outros aspectos que estão inseridos nas áreas de conhecimento abrangidas pela Engenharia de Produção. (BRASIL, 2010).

E no que se refere à educação do futuro Engenheiro de Produção, a Associação Brasileira de Engenharia de Produção (ABEPRO) entende que:

“Compete à engenharia de produção o projeto, a modelagem, a implantação, a operação, a manutenção e a melhoria de sistemas produtivos integrados de bens e serviços, envolvendo homens, recursos financeiros e materiais, tecnologia, informação e energia. Compete ainda especificar, prever e avaliar os resultados obtidos destes sistemas para a sociedade e o meio ambiente, recorrendo a conhecimentos especializados da matemática, física, ciências humanas e sociais, conjuntamente com os princípios e métodos de análise e projeto da engenharia" (ABEPRO, 2012).
Segundo Mello et al. (2011), para uma formação de qualidade é preciso que as instituições de ensino superior monitorem constantemente as competências demandadas pelo mercado. Isso porque, a ausência desse acompanhamento pode ter como resultado a adoção de um currículo com grande desequilíbrio, onde ocorra a priorização de determinados conhecimentos em detrimento de outros, ou um currículo extenso demais que pode tornar o curso mais longo, inviabilizando a sua continuidade para diversos estudantes.

Nesse sentido, o presente artigo visa analisar os conteúdos das provas do ENADE (Exame Nacional de Desempenho de Estudantes) do grupo VI de Engenharia, na busca de evidências sobre os conteúdos mais exigidos dos futuros profissionais da área de Engenharia de Produção. Além disso, apresentará a importância relativa das áreas da Engenharia de Produção para a avaliação de um egresso, posto que este exame é considerado uma valiosa ferramenta de avaliação educacional dos estudantes e das suas respectivas instituições de ensino superior em todo território nacional. Pode-se dizer, que o estudo tem por objetivo, ainda, subsidiar o aprimoramento dos cursos de Engenharia de Produção e de possibilitar ações específicas orientadas à preparação de discentes para o ENADE.

\section{Revisão da literatura}

\subsection{Engenharia de Produção}

A bibliografia de Taylor, principalmente a partir do livro Princípios de Administração Científica, foi uma das principais contribuições ao surgimento da Engenharia Industrial, que após algum tempo passou a ser denominada Engenharia de Produção.

Dentre as contribuições apresentadas neste livro destaca-se o estudo referente ao aumento da produtividade e a redução dos tempos e movimentos das atividades realizadas pelos operários. Tempos estes que Taylor considerava desnecessários, pois visava uma maior produtividade em menor tempo. $\mathrm{O}$ método empregado para a realização de tal estudo foi a utilização de um cronômetro para manter um controle sobre o trabalhador, evitando assim a sua ociosidade. 


\begin{abstract}
"Não há dúvida de que a tendência do homem comum, em todos os atos de sua vida, é trabalhar devagar e comodamente, é só depois de pensar e observar bem a esse respeito, ou como resultado de exemplo, consciência ou pressão exterior, ele adota um ritmo mais rápido" (TAYLOR, 1995: 27).
\end{abstract}

Posteriormente ao estudo de Taylor estes métodos foram utilizados em larga escala na indústria automobilística, por Henry Ford, que introduziu o sistema de "produção em massa". Dentro desse contexto e com o aprimoramento dos métodos empregados por Taylor e Ford criou-se uma base sólida para o surgimento do curso de Engenharia de Produção Industrial (Industrial Engineering para os americanos), posteriormente chamado Engenharia de Produção (Production Engineering para os ingleses).

Dessa forma, a Engenharia de Produção (EP) inciou-se em meados do século $\mathrm{XX}$ devido à necessidade de solucionar problemas nos processos fabris. De acordo com LEME (1983), no Brasil sua origem se deu na década de cinquenta, quando a Escola Politécnica da Universidade de São Paulo passou a oferecer o primeiro curso de EP.

Desde seu surgimento, o número de cursos existentes vem aumentando consideravelmente. Segundo OLIVEIRA et al. (2005), "a partir de 1998 houve um crescimento vertiginoso do número de cursos de Engenharia de Produção no Brasil, saltando dos trinta e oito registrados em 1997 para aproximadamente duzentos cursos em 2005, registrando-se a criação em torno de quase vinte cursos por ano". Uma consulta ao sistema e-MEC permite destacar que em 2012 já se registram 520 ofertas do curso.

\subsection{ENADE}

Em 1995, teve início no Brasil um processo gradual de implementação de um sistema de avaliação da educação superior que foi colocado em prática pelo governo Brasileiro com base na Lei 9.131, de 1995. Esta Lei estabeleceu a criação do Exame Nacional de Cursos (ENC), popularmente conhecido como Provão. Leis posteriores incluíram no sistema o Censo de Educação Superior e a Avaliação das Condições de Ensino (ACE), conforme destacam Dantas, Soares \& Verhine (2006).

Inicialmente, o Provão fora boicotado pelos estudantes, mas posteriormente, tornou-se parte da cultura da educação superior no Brasil. Apesar da sua evolução (de 3 áreas de conhecimento testadas em 1995 para 26 em 2003), o provão continuou recebendo críticas por parte de docentes, discentes e especialistas em avaliação, principalmente em relação a consistência e validade dos seus resultados (DANTAS, SOARES \& VERHINE, 2006; FERRAZ et al., 2009).

Frente a isso, a fim de aprimorar o sistema de avaliação do ensino superior, foi criada, em 2004, a Lei $\mathrm{n}^{\mathrm{o}}$. 10.861, que instituiu o Sistema Nacional de Avaliação da Educação Superior - SINAES com o "objetivo de assegurar o processo nacional de avaliação das instituições de educação superior, dos cursos de graduação e do desempenho acadêmico de seus estudantes".

A prova do ENADE, de acordo com o art. 1 da Lei 10.861 de 2004, tem como objetivo geral avaliar o desempenho dos estudantes em relação aos conteúdos programáticos previstos nas diretrizes curriculares, às habilidades e competências para a atualização permanente e aos conhecimentos sobre a realidade brasileira, mundial e sobre outras áreas do conhecimento.

A avaliação é composta por duas partes, sendo uma referente à Formação Geral do estudante - que é comum a todos os cursos de todas as áreas de conhecimento - e outra referente ao conhecimento específico adquirido ao longo de sua formação, que no caso da Engenharia de Produção está inserida na área de Engenharia - grupo VI. De acordo com o Brasil (2011), as questões específicas são divididas da seguinte forma: 3 discursivas e 27 de múltipla escolha, envolvendo situações-problemas e estudos de caso.

A Engenharia de Produção, que é o foco desse artigo, está inserida na área de Engenharia-Grupo VI, conforme destacado acima. E de acordo com o SINAES (2011), este grupo é composto ainda pelos seguintes cursos: Engenharia de Produção Mecânica, Engenharia de Produção Elétrica, Engenharia de Produção Química, Engenharia de Produção Têxtil, Engenharia de Produção de Materiais e Engenharia de Produção Civil. Ou seja, é composta pela Engenharia de Produção plena e pelas suas "ênfases".

Em relação a este ponto, Oliveira (2005) ressalta que inicialmente nos cursos tradicionais predominava a Engenharia de Produção como uma ênfase. Fato este possível através da inserção de "cadeiras" de Produção no curso de Engenharia Mecânica, por exemplo. Porém, a partir da década de 
90, os papéis se inverteram e começou a predominar os cursos com ênfase em bases tecnológicas tradicionais, como Engenharia de Produção com ênfase em Mecânica, por exemplo. De acordo com Batalha (2008), o objetivo da ênfase é fornecer uma formação que permita a atuação do profissional competente em diferentes setores de atividade, desta forma, serão fornecidas competências para a área tecnológica atrelada com competências da área de administração e gestão. Oliveira (2005) complementa que antes organizações industriais e empresariais tinham como base a capacidade de produção em escala, porém, atualmente os pilares sustentam-se no conhecimento (tecnológico e sistêmico) e na capacidade destas organizações em alcançar à melhoria constante dos seus produtos e processos de produção, que refletem nos seus principais diferenciais em termos de qualidade e produtividade.

\subsection{Comparação das áreas estabelecidas pelo ENADE e ABEPRO}

Existe uma "fronteira" entre o que o estudante aprende na sala de aula e o que realmente é exigido pelo mercado de trabalho. Isto se deve, segundo Pezzi (2005), à adoção de matrizes curriculares incoerentes e não alinhadas com o futuro campo de trabalho dos alunos por parte das instituições de ensino superior.

Nose \& Rebelatto (2001) acrescentam que esses conhecimentos que não foram vistos durante a vida acadêmica podem ser adquiridos pelo profissional ao longo de sua carreira, mas que de qualquer forma, os cursos deveriam ser estruturados de acordo com as necessidades da sociedade e do mercado de trabalho e não conforme conteúdos existentes.

Desta forma, o ENADE busca estabelecer as diretrizes das provas através da Comissão Nacional de Avaliação da Educação Superior (Conaes), órgão colegiado de coordenação e supervisão do Sistema Nacional de Avaliação da Educação Superior (Sinaes).

E de acordo com o Inep (2011), os conteúdos curriculares específicos da área do grupo VI são: Engenharia de Operações e Processos da Produção, Logística, Pesquisa Operacional, Engenharia da Qualidade, Engenharia do Produto, Engenharia Organizacional, Engenharia Econômica, Engenharia do Trabalho e Engenharia da Sustentabilidade.
Já a ABEPRO (2008) propõe que sejam lecionadas um total de dez áreas de conhecimento durante a graduação em Engenharia de Produção: Engenharia de Operações e Processos da Produção, Logística, Pesquisa Operacional, Engenharia da Qualidade, Engenharia do Produto, Engenharia Organizacional, Engenharia Econômica, Engenharia do Trabalho, Engenharia da Sustentabilidade e Educação em Engenharia de Produção. O quadro 1 ilustra os conteúdos específicos da área de Engenharia referente ao grupo VI segundo o ENADE (2011) e os conteúdos específicos de Engenharia de Produção de acordo ABEPRO (2008), fazendo uma relação das áreas equivalentes de cada uma.

Quadro 1 - Comparação entre as diretrizes do ENADE e da ABEPRO.

\begin{tabular}{|l|l|}
\hline ENADE (2011) & ABEPRO (2008) \\
\hline $\begin{array}{l}\text { Engenharia de } \\
\text { operações e processos } \\
\text { da produção }\end{array}$ & $\begin{array}{l}\text { Engenharia } \\
\text { operações processos } \\
\text { da produção }\end{array}$ \\
\hline Logística & Logística \\
\hline Pesquisa operacional & Pesquisa operacional \\
\hline Engenharia da qualidade & Engenharia da qualidade \\
\hline Engenharia do produto & Engenharia do produto \\
\hline $\begin{array}{l}\text { Engenharia } \\
\text { organizacional }\end{array}$ & $\begin{array}{l}\text { Engenharia } \\
\text { organizacional }\end{array}$ \\
\hline Engenharia econômica & Engenharia econômica \\
\hline Engenharia do trabalho & Engenharia do trabalho \\
\hline $\begin{array}{l}\text { Engenharia da } \\
\text { sustentabilidade }\end{array}$ & $\begin{array}{l}\text { Engenharia } \\
\text { sustentabilidade }\end{array}$ \\
\hline & $\begin{array}{l}\text { Educação em } \\
\text { Engenharia de produção }\end{array}$ \\
\hline
\end{tabular}

Através da análise do Quadro 1 pode-se concluir que as diretrizes do ENADE 2011 estão alinhadas à sugestão de áreas de conhecimento preconizadas pela ABEPRO e que há equivalência entre essas áreas, com exceção da área referente à Educação na Engenharia de Produção que não consta no ENADE como linha de conteúdo.

\section{Metodologia da pesquisa}

A princípio foi realizada uma revisão da literatura para a compreensão das competências e diretrizes cobradas nas provas específicas para a Engenharia grupo VI, além de outros pontos 
considerados importantes para a base teórica do presente estudo. De acordo com Vergara (2005) a pesquisa bibliográfica é o estudo sistematizado desenvolvido a partir de materiais publicados em livros, revistas, jornais e redes eletrônicas, isto é material acessível ao público em geral.

Quanto aos meios, a pesquisa deu-se através de artigos, livros, site do INEP (www.inep.com.br), manuais e editais publicados das provas do ENADE. $\mathrm{Na}$ segunda etapa, foi elaborada uma comparação entre as diretrizes que o ENADE apresenta em seu manual de 2011 e as áreas de conhecimento sugeridas pela ABEPRO (Associação Brasileira de Engenharia de Produção) em 2008.

"A pesquisa é uma atividade voltada para a investigação de problemas teóricos ou práticos por meio do emprego de processos científicos" (CERVO, 2007: 57).

Neste contexto, o presente estudo baseou-se no esquema ilustrado na figura 1 .

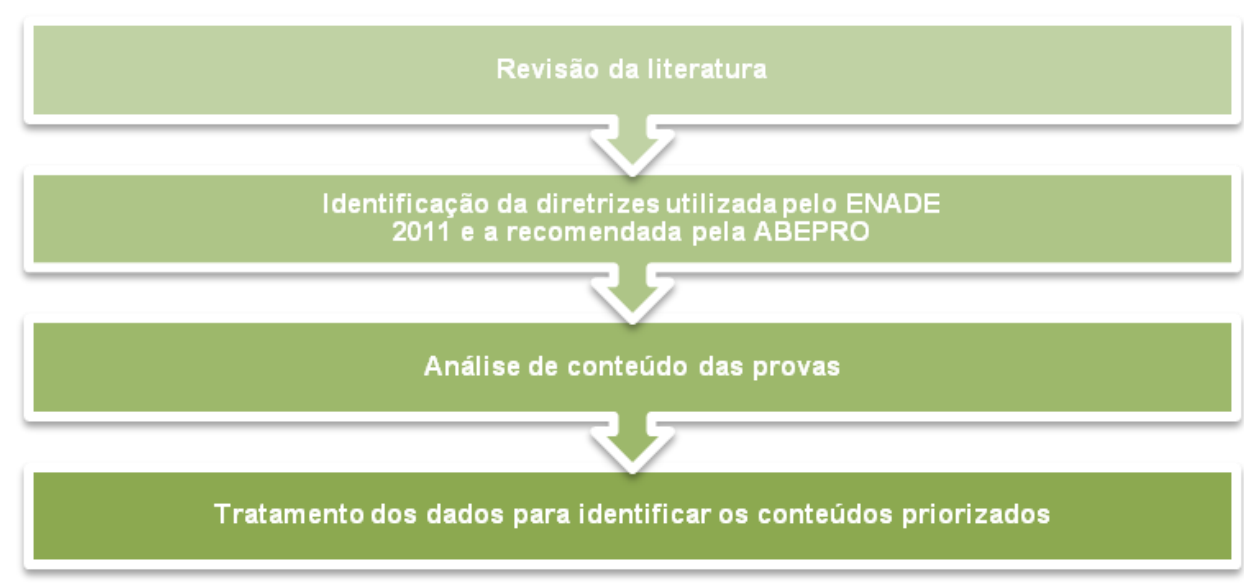

Figura 1- Etapas do Procedimento Metodológico da Pesquisa.

Após uma investigação teórica sobre o tema, foi realizado um estudo detalhado nas provas do ENADE especificas de Engenharia de Produção, dos anos de 2005, 2008 e 2011, compreendendo um total de 67 questões. Cada uma dessas questões foi categorizada segundo as áreas da Engenharia de Produção, e após o tratamento dos dados obtidos com a análise de todas as questões, identificou-se os conteúdos da Engenharia de Produção priorizados nas provas.

A limitação do método está na deficiência de não possuir um quantitativo maior de provas, posto que só houve possibilidade de análise de 3 provas do grupo VI de Engenharia. Visto que as provas da avaliação do ensino superior, atualmente conhecidas como ENADE, passaram a ser aplicadas somente a partir do ano de 2004, já que esta substituiu o chamado Provão que tinha a mesma função do sistema atual, porém com metodologia distinta.

Outro fator limitante encontra-se no fato de terem sido analisadas somente as provas específicas do grupo e não as de Formação Geral. Isso se justifica pela necessidade estabelecida de traçar alguma relação do conteúdo ENADE com as áreas de conhecimento definidas na diretriz da prova e também na orientação ABEPRO.

\section{Resultados da pesquisa}

\subsection{Identificação das Principais Áreas de Conhecimento}

A partir da apuração dos dados foi feita uma análise que possibilitou um entendimento mais reflexivo sobre as demandas de conteúdos projetadas aos graduandos do curso de Engenharia de Produção.

Sob uma percepção inicial é de se esperar que a área de Engenharia de Operações seja classificada como a principal área de conhecimento da engenharia de produção. Portanto, a contabilização e identificação da representatividade de cada área possibilita uma visualização quantitativa global que demonstre como os conteúdos foram cobrados nos três anos analisados, 2005-2008-2011. O gráfico 1, a seguir, ilustra a representatividade das áreas de conhecimento perante a totalidade das questões consideradas para efeito da presente pesquisa. 


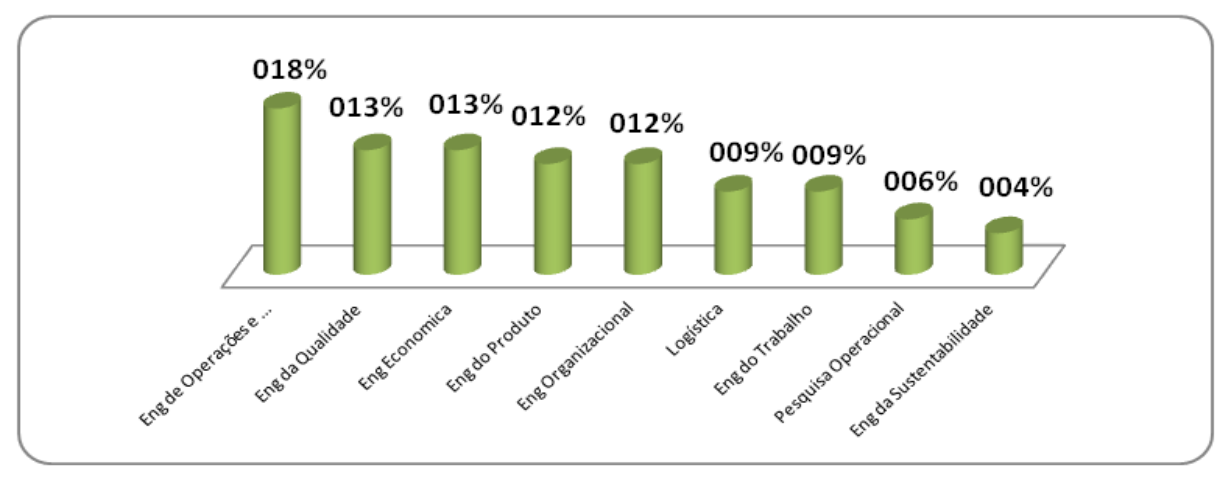

Gráfico 1- Representatividade das áreas de conhecimento.

O gráfico 1, ilustra o quantitativo percentual das 67 (sessenta e sete) questões das provas aplicadas pelo ENADE ao longo destes três anos. Com base nesses elementos foi possível depreender as 03 (três) áreas que foram mais intensamente abordadas durante este período: Engenharia de Operações e Processos (com 17,91\% das questões), Engenharia da Qualidade e Engenharia Econômica (ambas com 13,43\%).

Estas três áreas totalizam $44,78 \%$ das questões analisadas no referido intervalo de tempo, conteúdos considerados fundamentais para a formação de um Engenheiro de Produção, o qual é considerado um profissional dotado de conhecimentos técnicos e de gestão, e por conseguinte, capacitado para atuar em distintas áreas organizacionais.

Observa-se que a área de Engenharia de Operações e Processos foi a que obteve um maior percentual de questões com $17,91 \%$ do total. Tal

Tabela 1- Percentual de questões Área/Ano resultado pode ser explicado pela visão que as bancas organizadoras de tais provas têm de um curso de Engenharia de Produção. Ao enfatizar tais conteúdos, destaca-se a área de Engenharia de Operações e Processos como uma das mais importantes, a qual de acordo com a ABEPRO (2008), engloba importantes disciplinas como: Gestão de Sistemas de Produção e Operações, Planejamento, Programação e Controle da Produção, Gestão da Manutenção, Projeto de Fábrica e de Instalações Industriais: organização industrial, layout/arranjo físico, Processos Produtivos Discretos e Contínuos: procedimentos, métodos e sequencias e Engenharia de Métodos.

A fim de uma análise mais refinada de cada ano formulou-se a tabela 1, a seguir, em que trata o percentual de questões distribuídas ao longo do referido intervalo.

\begin{tabular}{|lcccc|}
\hline \multirow{2}{*}{ Áreas selecionadas } & \multicolumn{4}{c|}{ Anos (valores percentuais) } \\
\cline { 2 - 5 } & $\mathbf{2 0 0 5}$ & $\mathbf{2 0 0 8}$ & $\mathbf{2 0 1 1}$ & Total de questões por área \\
\hline Engenharia Econômica & $10,0 \%$ & $15,0 \%$ & $14,8 \%$ & 9 \\
\hline Engenharia de Operações e Processos & $25,0 \%$ & $20,0 \%$ & $11,1 \%$ & 12 \\
\hline Engenharia da Qualidade & $10,0 \%$ & $15,0 \%$ & $14,8 \%$ & 9 \\
\hline Engenharia Organizacional & $15,0 \%$ & $5,0 \%$ & $14,8 \%$ & 8 \\
\hline Engenharia do Produto & $10,0 \%$ & $10,0 \%$ & $14,8 \%$ & 8 \\
\hline Pesquisa Operacional & $10,0 \%$ & $5,0 \%$ & $3,7 \%$ & 4 \\
\hline Logística & $5,0 \%$ & $15,0 \%$ & $14,8 \%$ & 8 \\
\hline Engenharia do Trabalho & $10,0 \%$ & $10,0 \%$ & $7,4 \%$ & 6 \\
\hline Engenharia da Sustentabilidade & $5,0 \%$ & $5,0 \%$ & $3,7 \%$ & 3 \\
\hline Total & $\mathbf{1 0 0 , 0 \%}$ & $\mathbf{1 0 0} \%$ & $\mathbf{1 0 0 \%}$ & $\mathbf{6 7}$ \\
\hline Total/Ano & $\mathbf{2 0}$ & $\mathbf{2 0}$ & $\mathbf{2 7}$ & \\
\hline
\end{tabular}


De acordo com o exposto na tabela 1, pode-se perceber o baixo índice de questões que abordam a área de Engenharia da Sustentabilidade, com $5 \%$ em 2005, 5\% em 2008 e 5\% em 2011 das questões; correspondendo um total de apenas 3 questões, uma em cada ano. Embora o tema sustentabilidade seja atual e amplamente discutido nas bases educacionais o referido exame tem atribuído o mesmo peso desde sua criação. Em outro tipo de análise é possível perceber que os conteúdos de Engenharia Organizacional foram utilizados de forma intensa na primeira edição da prova. O gráfico 2 , abaixo, foi construído realizando a confrontação anos/áreas.

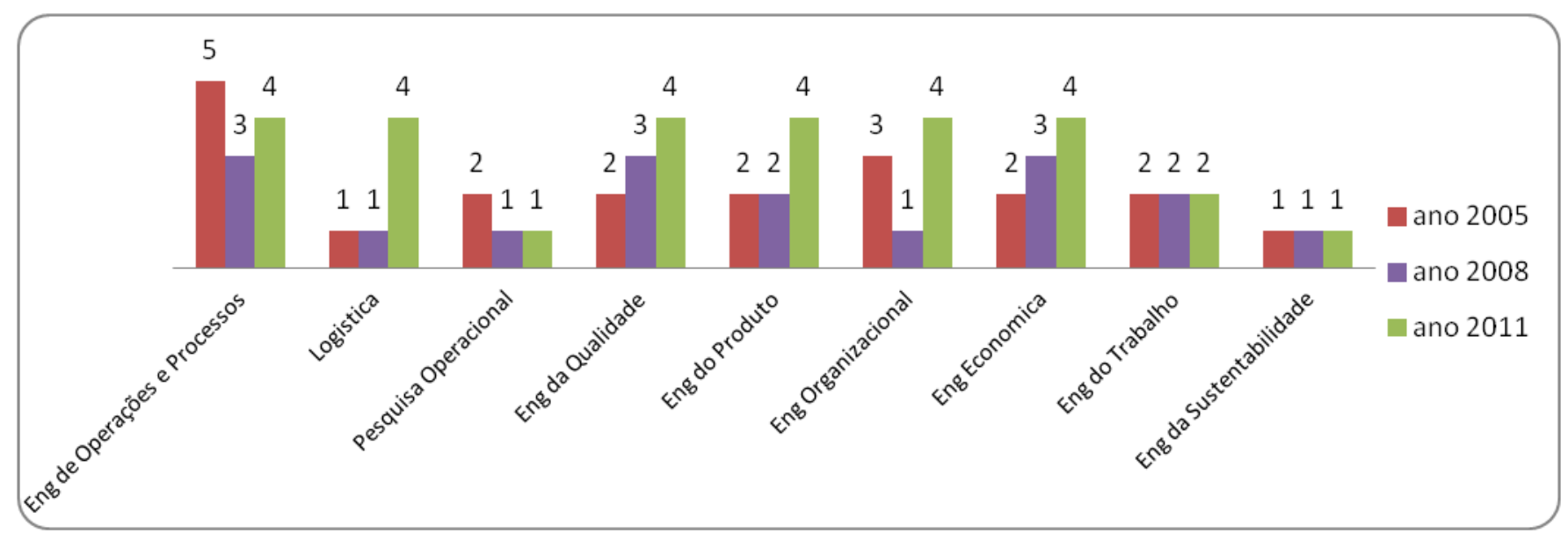

Gráfico 2- Quantitativo de questões das áreas relacionadas ao longo dos anos.

Em 2011 nota-se um aumento do quantitativo de questões em relação aos dois anos anteriores, fato este já explicitado na tabela 2 , posto que o número de questões elevou-se de 20 (anos 2005 e 2008) para 27 (ano 2011). Outro ponto a ser ressaltado em relação à evolução ao longo dos anos é a mudança da abordagem de cada questão, visto que a princípio as provas eram mais objetivas e diretas ao passo que, atualmente uma única questão pode abordar vários conteúdos enfatizando assim a característica de multidisciplinaridade.

No que tange ao desenvolvimento das áreas, nota-se que o número de questões da área de Logística elevou-se de apenas uma questão em 2005 para quatro em 2011. Em contraponto, na área Engenharia de operações e processos ocorreu uma queda passando de cinco questões em 2005 para três questões em 2011.

No ano de 2011, constatou-se a linearidade da distribuição das áreas de Logística, Engenharia da Qualidade, Engenharia do Produto, Engenharia Organizacional e Engenharia Econômica.

Durante a análise das provas além da divisão das questões por área, foi possível destacar a cada área de conhecimento determinados conteúdos disciplinares. Desta forma, os itens relacionados, abaixo, destacam alguns temas das áreas sugeridas pelo ENADE (2011) em ordem decrescente de representatividade (conforme ilustra o gráfico 1):

a) Engenharia de Operações e Processos: tipos de layout, tempo de processamento, taxa de produção média, posto de trabalho gargalo, aumento da produtividade, tempos, projeto no processo de trabalho, tipos de processo e capacidade produtiva.

b) Engenharia da Qualidade: gestão da qualidade total, controle estatístico do processo, gráfico de controle e sistema de gestão integrada.

c) Engenharia Econômica: viabilidade econômica, ponto de equilíbrio, viabilidade de projeto, VPL, custeio ABC e FIFO.

d) Engenharia do Produto: ciclo de vida do produto, engenharia simultânea, sustentabilidade, ecodesign, gestão do desenvolvimento do produto (GDP) e desdobramento da função qualidade (QFD).

e) Engenharia Organizacional: estratégia, ações estratégicas, sistema integrado de gestão, multifuncionalidade, tecnologia da informação, inovação e intercâmbio de dados. 
f) Logísitca: cadeia de suprimentos, lote econômico, logítica reversa e arranjos produtivos locais (APL).

g) Engenharia do Trabalho: tempos e movimentos, tarefas, fatores ergonômicos e antropométricos, postos de trabalho e acidentes de trabalho.

h) Pesquisa Operacional: Pert, programação linear e otimização.

i) Engenharia da Sustentabilidade: processo de produção, sustentabilidade e impacto ambiental.

Vale ressaltar que alguns temas a princípio podem parecer terem sido alocados de forma errônea, porém tal fator é explicado pelo caráter multidisciplinar que o referido exame tem assumido recentemente. Visto que uma única questão pode englobar várias disciplinas, optou-se por tabulá-la atrelada à disciplina que fosse considerada primordial para a solução da questão considerada.

\section{Conclusões e sugestões de estudos futuros}

O presente artigo buscou destacar quais são as áreas de conteúdo mais importantes e exigidas de um graduando de Engenharia de Produção, segundo a visão dos organizadores das provas do ENADE.

Diferente do que fora evidenciado por Mello et al. (2011) que, após a análise aprofundada de um total de 24 provas (743 questões) de diversos concursos de acesso a organizações públicas, conseguiu identificar 5 áreas de conhecimento mais exigidas, dada a exígua diferença entre objetivos a que se propõe cada prova, foi possível observar que no ENADE ocorre um equilíbrio entre as mesmas. $\mathrm{O}$ qual evidencia a sua pretensão em avaliar de forma geral se os graduandos conseguiram absorver os conteúdos programáticos considerados fundamentais para sua formação.

Vale destacar que através da divulgação dos resultados do ENADE é possível que instituições utilizem como modelo educacional cursos que obtiveram uma boa avaliação no exame, buscando desta maneira o desenvolvimento do ensino superior brasileiro e a melhoria da formação desses futuros profissionais que atenderão ao mercado de trabalho.

Contudo, Oliveira (2005) ressalta que para os cursos evoluírem não basta que se invista no aprimoramento de seus currículos e de suas instalações, há que se investir em melhorias organizacionais e de processos de ensino/aprendizagem que possam apresentar melhores métodos e meios educacionais. E através do sistema de avaliação do ensino superior são constados os problemas da instituição e apontadas as melhorias, desta forma, o exame não deve ser considerado apenas um "ranking" em que serão apontadas as melhores e as piores, mas deve ser considerado um sistema para fazer progredir e implantar melhorias nos cursos.

Em relação a estudos futuros, sugere-se uma investigação mais profunda acerca das diferenças entre as provas de acesso a organizações públicas e as provas de avaliação do exame ENADE.

\section{Referências}

ABEPRO (2008). Documento Elaborado pela Comissão de Graduação e referendado no GT de Graduação do Encep 08 e Enegep 08 - 16/10/08.

ABEPRO (2012) - Associação Brasileira de Engenharia de Produção, <www.abepro.org.br> Acessado em: 4 de Abril de 2012.

BATALHA, M. O.; et al. Intodução à Engenharia de Produção. Rio de Janeiroo: Elsevier, 2008. $3^{\text {o }}$ reimpressão. 3 p.

BRASIL. Lei no. 10.861 de 14 de abril de 2004. Institui o Sistema Nacional de Avaliação da Educação Superior (SINAES). Brasília, 2004.

BRASIL. Lei no. 9.131 de 24 de novembro de 1995. Altera dispositivos da Lei $n^{\circ}$. 4.024, de 20 de dezembro de 1961 e dá outras providências. Brasília, 24 nov. 1995. Disponível em: <http://www.planalto.gov.br/ccivil_03/leis/L9131.ht m >. Acessado em: 25 de Abril de 2012.

CERVO, Amando Luiz; BERVIAN, Pedro Alcino \& SILVA, Roberto da.. Metodologia Cientifica. 6. Ed.. São Paulo: Pearson Prentice Hall, 2007. 159 p.

DANTAS, L.M.V. ; SOARES, J.F. \& VERHINE, R.E..Do Provão ao ENADE: uma análise comparativa dos exames nacionais utilizados no Ensino Superior Brasileiro. Revista Ensaio: Avaliação e Políticas Públicas em Educação, Rio de Janeiro, Vol. 14 n. 52, p. 291-310, 2006. 
e-MEC. Instituições de Educação Superior e Cursos Cadastrados. < http://emec.mec.gov.br>. Acessado em 17 de Dezembro de 2012.

FERRAZ, T. C. P.; GRASSANO, D. R.; LIMA, L. F. \& OLIVEIRA, V. F.. Um estudo das competências trabalhadas no ENADE 2005 nos cursos de Engenharia de Produção. XXIX ENCONTRO NACIONAL DE ENGENHARIA DE PRODUÇÃO. A Engenharia de Produção e o Desenvolvimento Sustentável: Integrando Tecnologia e Gestão. Salvador, BA, Brasil, 06 a 09 de outubro de 2009.

INEP. Instituto Nacional de Estudos e Pesquisas Educacionais Anísio Teixeira; Conselho Federal de Engenharia, Arquitetura e Agronomia, Brasília: 2010.

INEP. Portaria Inep no 245 de 04 de agosto de 2011. Publicada no Diário Oficial de 05 de agosto de 2011, Seção 1, págs. 55 e 56. Brasília, 2011.

LEME, R. A. S. A história da engenharia de produção no Brasil. In: ENCONTRO NACIONAL DE ENGENHARIA DE PRODUÇÃO, 3, São Paulo. Anais... São Paulo, 1983.

MELLO, J.A.V.B.; MIRANDA, L. K.; MELLO, A.J.R.; SILVA, D. M. \& SOUSA, F. R. Caminhos formacionais na engenharia de produção: evidências sobre demandas das organizações públicas. Anais do III Encontro Fluminense de Engenharia de Produção. Rio de Janeiro/ RJ, 10 e 11 de Novembro de 2011.

NOSE, M. M.; REBELATTO, D. A. do N. .O perfil do Engenheiro segundo as empresas. Cobeng. 2001.

OLIVEIRA, V. F.. A avaliação dos cursos de engenharia de produção. Gestão Industrial. v. 01, n. 03 : pp.001-012, 2005.

OLIVEIRA, V. F.; BARBOSA C. S. \& CHRISPIM E. M. Cursos de Engenharia de Produção no Brasil: Crescimento e Projeções. Anais do XXV Encontro Nac. de Engenharia de Produção. Porto Alegre, RS, Brasil, 29 out a 01 de Novembro de 2005.

PEZZI, M. R.; MEDEIROS, R.. Engenharia de Produção: Limites e Possibilidades Curriculares.
XII SIMPEP- Bauru, SP, Brasil, 7 a 9 de Novembro de 2005

SINAES (Sistema Nacional de Avaliação de Educação Superior). Seminários do ENADE 2011Questões operacionais. 2011. <http://portal.inep.gov.br/seminarios-enade>.

Acessado em: 4 de Abril de 2012.

TAYLOR, F. W.. Princípios de Administração Científica. São Paulo: Atlas, 1995.

VERGARA, S. C. Métodos de pesquisa em administração. São Paulo: Atlas, 2005.
Artigo selecionado entre os 10 melhores do VIII Encontro Mineiro de Engenharia de Produção - EMEPRO 2012. 